\title{
P1084: IMPACT OF A CLINICAL PATHWAY IN THE MANAGEMENT OF HYPERGLYCEMIC CRISES IN A PRIVATE TERTIARY HOSPITAL IN THE \\ PHILIPPINES
}

Ma. Karen Celine C. Ilagan, MD-MBA, Karen Elouie C. Agoncillo, MD, Perie A. Wagan, MD, Ruben G. Kasala, MD - Section of Endocrinology, Diabetes \& Metabolism, The Medical City



\section{BACKGROUND}

- The use of clinical pathways in the management of diabetes emergencies has been shown to improve clinical outcomes.

- In line with this, The Medical City (TMC) implemented the Clinical Pathway for Hyperglycemic Crises in 2010.

- Six years after its implementation, a retrospective cross-sectional study comparing the clinical and safety outcomes of pathway-managed versus non-pathway-managed diabetic patients with diabetic ketoacidosis (DKA) or hyperglycemic hyperosmolar state (HHS) admitted to TMC was completed.

\section{OBJECTIVES}

To compare the clinical and safety outcomes of the TMC Clinical Pathway for Hyperglycemic Crises from non-pathway management among DKA and HHS patients admitted from January 2010 to April 2016.

\section{METHODS}

- Retrospective cross-sectional comparative study

- Review of electronic medical records (EMR)

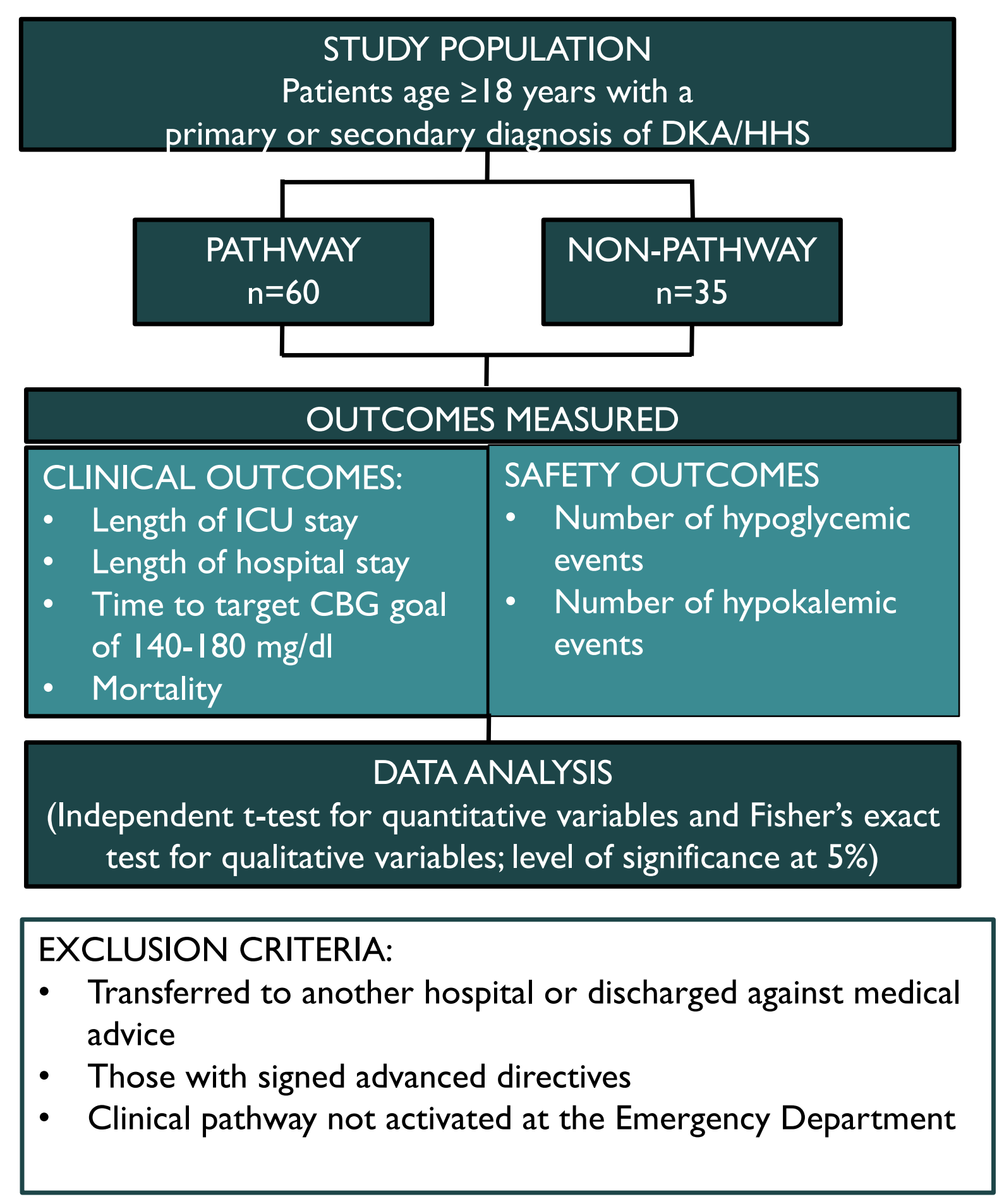

\section{RESULTS}

Table 1. Baseline diagnostics on admission.

\begin{tabular}{|l|c|c|c|}
\hline & $\begin{array}{c}\text { PATHWAY } \\
\mathbf{n = 6 0}\end{array}$ & $\begin{array}{c}\text { NON- } \\
\text { PATHWAY } \\
\mathbf{n = 3 5}\end{array}$ & P VALUE \\
\hline HbAlc & $11.3 \pm 0.5$ & $9.4 \pm 0.8$ & $\mathbf{0 . 0 3 9}$ \\
\hline Admission CBG & $467 \pm 24.3$ & $429 \pm 26.2$ & 0.312 \\
\hline Arterial pH & $7.22 \pm 0.1$ & $7.09 \pm 0.2$ & 0.402 \\
\hline Ser Osmolality & $310.1 \pm 9.1$ & $299.5 \pm 4.6$ & 0.399 \\
\hline Potassium & $4.67 \pm 0.1$ & $4.07 \pm 0.2$ & $\mathbf{0 . 0 0 4}$ \\
\hline Creatinine & $1.21 \pm 0.1$ & $1.80 \pm 0.3$ & 0.050 \\
\hline Ser Ketones & $1.53 \pm 0.1$ & $1.14 \pm 0.1$ & $\mathbf{0 . 0 1 2}$ \\
\hline Ser Bicarbonate & $12.6 \pm 0.9$ & $14.1 \pm 1.0$ & 0.326 \\
\hline
\end{tabular}

Figure 1. Comparison of Clinical Outcomes.



LENGTH OF HOSPITAL LENGTH OF ICU STAY STAY (DAYS)

(DAYS)

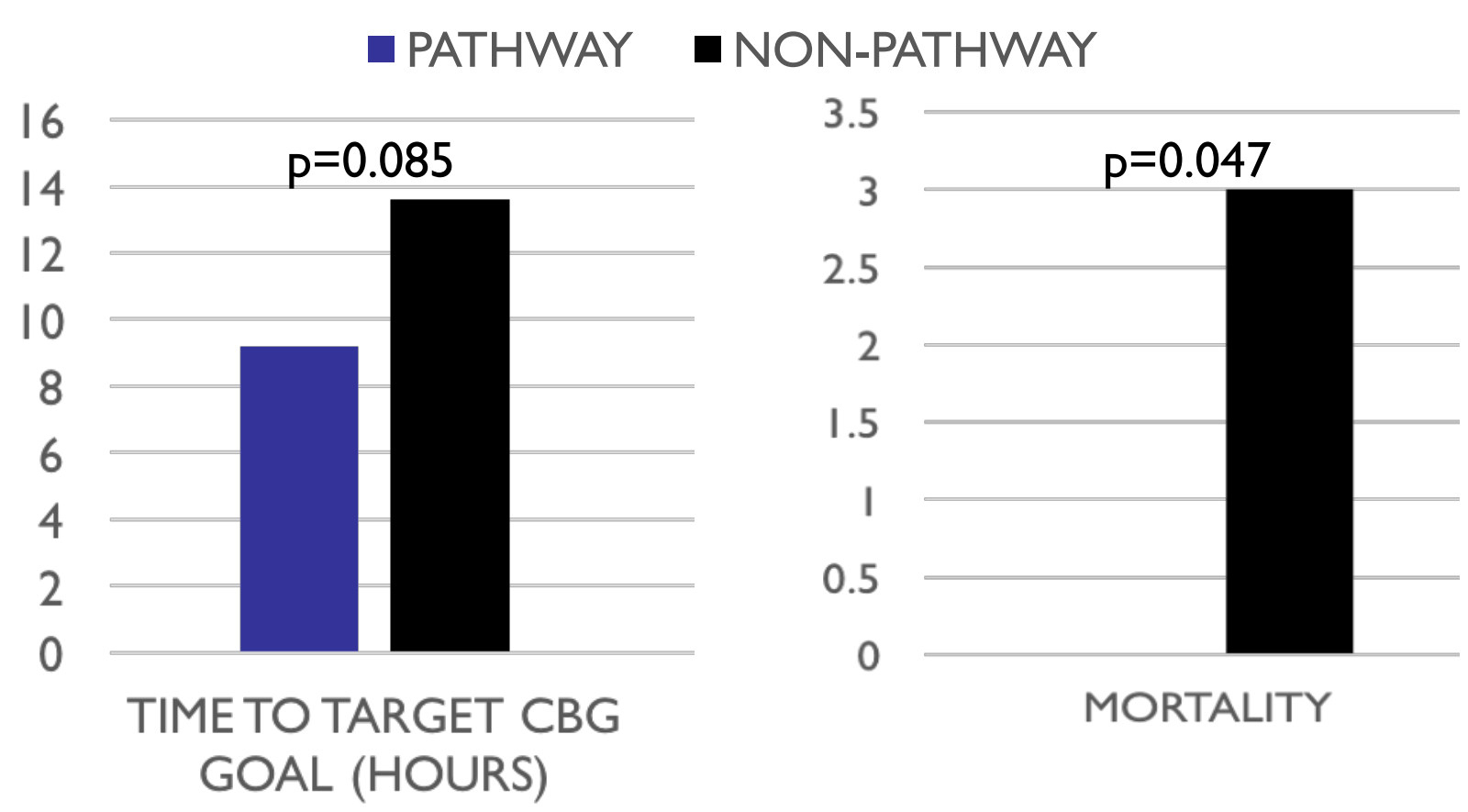

Figure 2. Comparison of Safety Outcomes.

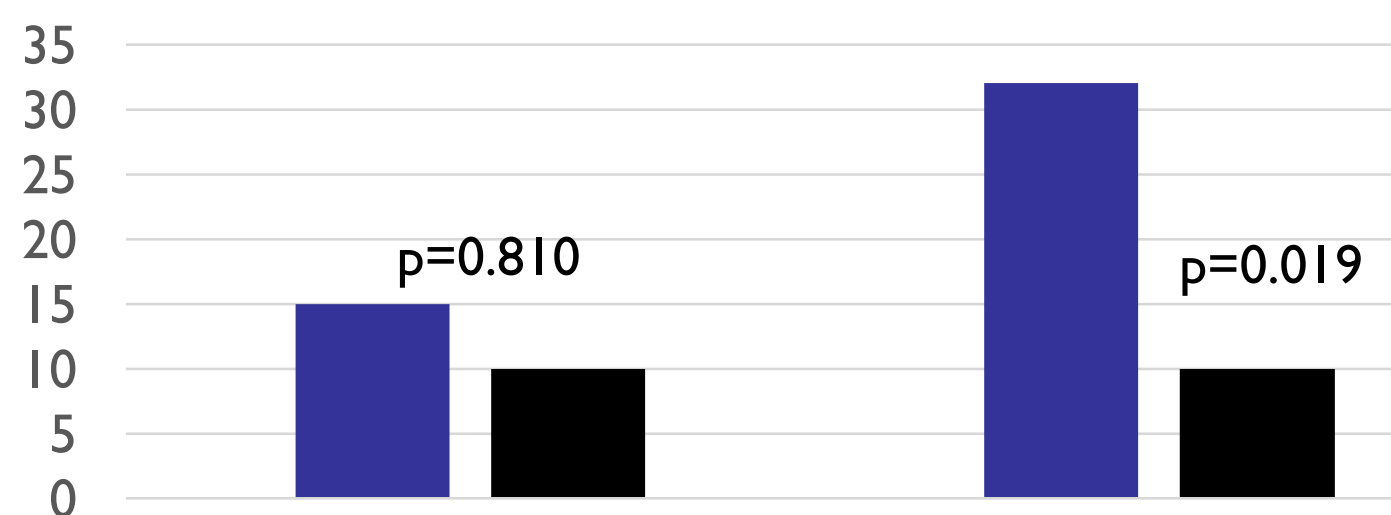

NO. OF HYPOGLYCEMIC NO. OF HYPOKALEMIC EVENTS EVENTS

\section{CONCLUSIONS \& RECOMMENDATIONS}

DKA and HHS patients managed using a clinical pathway had lower mortality rates compared to those managed without a pathway. However, there was an increase in hypokalemic events which were immediately corrected in the pathway group.

- Both groups were similar in terms of time to reach target CBG goal, hypoglycemic events, length of hospital stay, and length of ICU stay.

- Implementation of the TMC pathway should be continued.

- Further studies with larger sample size, a prospective nature,, determination of adherence to pathway, and cost-benefit analysis.

\section{REFERENCES}

Kittabachi A, Miles J, Umpierrez G, and Fisher J. (2009). Hyperglycemic Crises in Adult Patients. Diabetes Care, 1335-1343

Hara JS, Rahbar AJ, Jeffres MN, and Izuora KE. (20I3). Impact of a Hyperglycemic Crises Protocol. Endocrine Practice.

Singh, RK, P Perros, and BM Frier. (1997). Hospital Management of Diabetic Ketoacidosis:Are Clinical Guidelines Implemented Effectively? Diabetic Medicine. 14: 482 - 486. 